\title{
Testing the Unified Model with an Infrared Selected Sample of Seyferts
}

\author{
H. R. Schmitt and J. S. Ulvestad \\ National Radio Astronomy Observatory, P.O. Box 0, Socorro, NM87801
}

R. R. J. Antonucci

University of California, Santa Barbara, Santa Barbara, CA93106

C. J. Clarke and J. E. Pringle

Institute of Astronomy, Madingley Road, Cambridge CB3 OHA, England

\author{
A. L. Kinney
}

NASA Headquarters, 300 E St., Washington, DC20546

\begin{abstract}
.
We present a series of statistical tests using homogeneous data and measurements for a sample of Seyfert galaxies. These galaxies were selected from mostly isotropic properties, their far infrared fluxes and warm infrared colors, which provide a considerable advantage over the criteria used by most investigators in the past, like ultraviolet excess. Our results provide strong support for a Unified Model in which Seyferts 2's contain a torus seen more edge-on than in Seyferts 1's and show that previous results showing the opposite were most likely due to selection effects.
\end{abstract}

\section{Introduction}

The Unified Scheme is based on the idea that the nucleus is surrounded by a dusty molecular torus, with orientation angle being the parameter which determines whether an AGN is perceived by observers as a Seyfert 1 or as a Seyfert 2 (Antonucci 1993). This scenario is supported by the detection of polarized broad emission lines in Seyfert 2's (Antonucci \& Miller 1985) and the collimated escape of radiation from nuclear region, detected as Narrow Line Regions with conical shapes in Seyfert 2 galaxies (Pogge 1989) and jet like radio emission (Ulvestad \& Wilson 1989).

It is now accepted that the Unified Model applies to a large fraction of Seyfert galaxies and is correct to first order. However, some observational results claim intrinsic statistical differences between Seyfert 1's and Seyfert 2's. Malkan et al. (1998) found that Seyfert 1's usually reside in earlier type host galaxies compared to Seyfert 2's, while Laurikainen \& Salo (1995) and Dultzin-Hacian 
et al. (1999) found a higher percentage of companions around Seyfert 2's than in Seyfert 1's.

We believe that these differences between Seyfert 1's and Seyfert 2's are mostly due to the way these papers selected their samples. In order to be able to make a fair comparison between Seyfert 1's and Seyfert 2's and correctly address problems related to the Unified Model, it is necessary to use a sample selected from an isotropic property, believed to be independent of the orientation of the torus relative to the line of sight.

\section{Sample and Data}

One of the best ways to select an isotropic sample is based on the far infrared properties of the galaxies. According to the Pier \& Krolik (1992) torus models, the circumnuclear torus radiates nearly isotropically at $60 \mu \mathrm{m}$, so a sample selected in this way should be relatively free from selection effects.

Our sample was extracted from the survey of warm Seyfert galaxies defined by de Grijp et al. (1992), which was selected based on the strength of their IRAS $25 \mu \mathrm{m}$ and $60 \mu \mathrm{m}$ fluxes and warm infrared colors. We selected all Seyfert galaxies with $\mathrm{z} \leq 0.031$, which gives a total of 29 Seyfert 1's and 59 Seyfert 2's. We have to point out, however, that this sample is not complete, since any Seyfert galaxy with infrared colors cooler than our criteria or for which there is no good quality IRAS data, are missed.

Another important point in this study is the use of high quality radio and optical images, obtained and measured homogeneously. We use broadband B and I images obtained for all the galaxies by Schmitt \& Kinney (2000), which will be used to determine host galaxy inclinations and, in some cases, morphological types. We also used VLA A-configuration $3.6 \mathrm{~cm}$ continuum images for 75 of the galaxies (Schmitt et al. 2001a), which will be used to determine the $3.6 \mathrm{~cm}$ radio powers and the extent of the radio emission in these galaxies.

\section{Infrared and Radio Luminosities}

The left panel of Figure 1 presents the distribution of $60 \mu \mathrm{m}$ luminosities for Seyfert 1's and Seyfert 2's. We can see that they have very similar distributions, with the Kolmogorov-Smirnov test (KS-test) giving a $45 \%$ chance that two samples drawn from the same parent population would differ this much. This result is expected since the galaxies were selected by their infrared properties and shows that this measurement is isotropic.

The distribution of $3.6 \mathrm{~cm}$ radio powers is presented on the right panel of Figure 1. Both Seyfert types have similar radio power distributions, as expected from the Unified Model, with the KS test showing that two samples selected from the same parent population would differ this much $11 \%$ of the time.

\section{Host Galaxy Properties}

The left panel of Figure 2 shows the observed distribution of the ratio between the host galaxies minor to major axis lengths $(\mathrm{b} / \mathrm{a})$. The observed distribution 

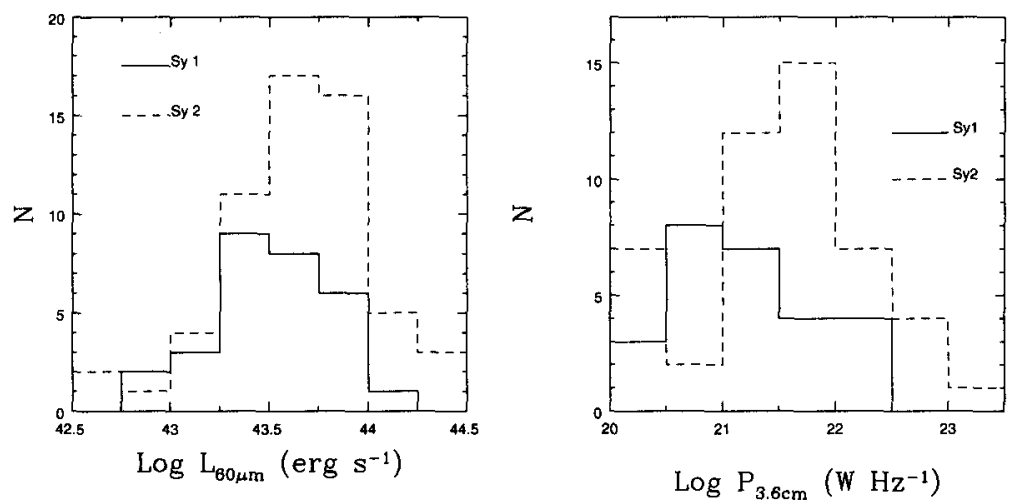

Figure 1. Left: histogram of the $60 \mu \mathrm{m}$ luminosity; right: histogram of the radio continuum $3.6 \mathrm{~cm}$ power. Seyfert 1's and Seyfert 2's are represented by solid and dashed lines, respectively.

has a deficit of Seyfert 1 galaxies with $b / a<0.5$, while in Seyfert 2's this does not happen. The lack of galaxies with $b / a<0.2$ is due to the thickness of the disk. Comparing the b/a distribution for Seyfert 1's and Seyfert 2's we find that they are significantly different, with the KS test giving the probability that two samples drawn from the same parent population would differ this much only $4.7 \%$ of the time.

This result is similar to the one found by Keel (1980), who was the first to discover a deficiency of edge-on Seyfert 1 galaxies (see also Lawrence \& Elvis 1982 and Maiolino \& Rieke 1995). Although this result is in principle not expected from the Unified Model, it does not necessarily contradict it. The papers cited above suggested that, in the case of edge-on Seyfert galaxies, the gas and dust along the host galaxy disk can block the direct view of the Broad Line Region, thus leading to a classification as a Seyfert 2 galaxy.

The comparison between the Morphological Types of the host galaxies of Seyfert 1's and Seyfert 2's is shown on the right panel of Figure 2. The two distributions are very similar, with the KS test showing that two samples selected from the same parent population would differ this much $80 \%$ of the time.

\section{Radio Sizes}

The distributions of the logarithm of the extension of the $3.6 \mathrm{~cm}$ radio emission in Seyfert 1's and Seyfert 2's is presented in Figure 3. Only 9 out of 26 Seyfert 1 's $(35 \%)$ show extended emission, and the rest are unresolved, as indicated by arrows in the figure. In the case of Seyfert 2's, 28 out of 48 galaxies (58\%) have extended radio emission.

Given the fact that $50 \%$ of the galaxies in our sample are unresolved, we had to use survival analysis to compare the two distributions. The mean and standard deviations of the extension of the radio emission were calculated using the Kaplan-Meier estimator, which gave $148 \pm 65 \mathrm{pc}$ and $348 \pm 97 \mathrm{pc}$ for Seyfert 1's 


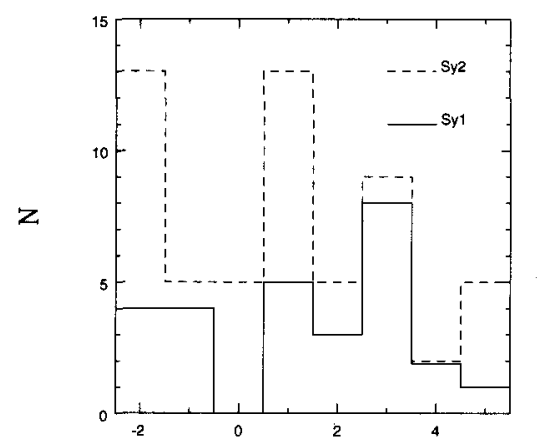

Morphological Type (T)

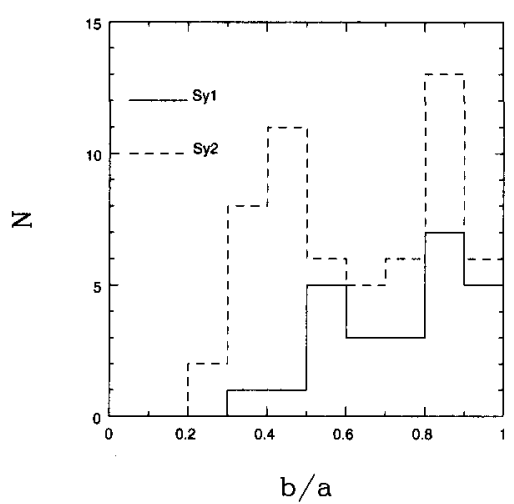

$\mathrm{b} / \mathrm{a}$

Figure 2. Histograms of morphological types, left, and the ratio between the semiminor and semimajor axes of the host galaxies, right.

and Seyfert 2's, respectively. We also compared whether Seyfert 1's and Seyfert 2's have similar distributions of the extension of the radio emission, using the Gehan-Wilcoxon test. We obtained a probability of $4.2 \%$ that the two samples are drawn from the same parent population. This confirms that Seyfert 1's have smaller extended emission, as predicted by the Model.

\section{Frequency of Companions}

Several mechanisms have been suggested to explain how to transport gas from the disk of a spiral galaxy to its nucleus, like interactions (Gunn 1979; Hernquist 1989) or bars (Schwartz 1981). The influence of interactions on the fueling of AGN has been the topic of several papers, but so far there is no consensus about this subject. Dahari (1984) and Rafanelli et al. (1995), among others, found Seyferts to have an excess of companions relative to normal galaxies. On the other hand, Fuentes-Williams \& Stocke (1988) and Bushouse (1986) found that there is no detectable difference in the environments of Seyfert and normal galaxies. An intriguing result was obtained by Laurikainen \& Salo (1995) and Dultzin-Hacyan et al. (1999), who showed that Seyfert 2's have a larger number of companions when compared to normal galaxies, while Seyfert 1's do not.

We used our broad band images, NED and the Digitized Sky Survey plates (DSS) to search for companions around our galaxies, and adopted the parameters used by Rafanelli et al. (1995) to determine if a galaxy has a companions. A galaxy is considered a companion if its distance to the galaxy of interest is smaller than 3 times the diameter of that galaxy (3D), the difference in brightness between them is smaller than 3 magnitudes $(|\Delta m| \leq 3 \mathrm{mag})$ and the radial velocities difference is smaller than $|c \Delta z| \leq 1000 \mathrm{~km} \mathrm{~s}^{-1}$.

According to these criteria, a total of 25 out of the 88 Seyfert galaxies in our sample have galaxies with $|\Delta I|$ or $|\Delta B| \leq 3 \mathrm{mag}$ and closer than 3D from them, which puts an upper limit of $<28 \pm 6 \%$ of possible interacting galaxies in this sample (the uncertainty is given by Poisson statistics). Of these 25 galaxies, 


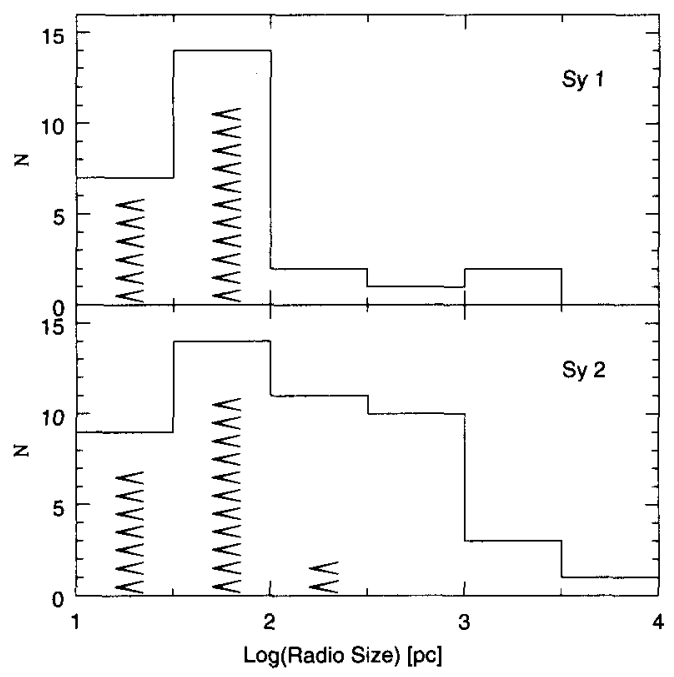

Figure 3. The distribution of the logarithm of the extension of the radio emission in Seyfert 1's and Seyfert 2's. The histograms represent the total number of galaxies in each bin, adding those with detected extended emission and upper limits, represent by arrows.

9 are Seyfert 1's and 16 are Seyfert 2's, which gives an upper limit of possible companions of $<31 \pm 10 \%$ and $<27 \pm 7 \%$, respectively. When we consider only the galaxies which satisfy the brightness, distance and velocity criteria, we find 17 Seyferts with confirmed companions. Since there is no information on the radial velocities for 8 of the possible companion galaxies, we assume that 17 is a lower limit, which gives that the percentage of Seyferts with companions is $>19 \pm 5 \%$. Of these 17 galaxies, 7 are Seyfert 1's and 10 are Seyfert 2's, corresponding to a lower limit of $>24 \pm 9 \%$ and $>17 \pm 5 \%$ galaxies with companions, respectively.

The percentage of confirmed companions in our sample is similar to the one found by Rafanelli et al. (1995) for the CfA sample, and also to the ones obtained by Schmitt (2001) for the Palomar sample, which is the same as for galaxies with other activity types. We should also notice that there is no apparent difference in the upper and lower percentage of companion galaxies in Seyfert 1's and Seyfert 2's, contradicting the results obtained by Laurikainen \& Salo (1995) and Dultzin-Hacyan et al. (1999). An explanation why these paper got to their results is given by Schmitt et al. (2001b).

\section{Summary}

We presented a series of test to the Unified Model of Seyfert galaxies based on a sample of galaxies selected by their infrared properties, which presents several advantages relative to other samples. The far infrared is a mostly isotropic property, which is essential for testing Unified Models, since the sample is unbiased 
relative to the orientation of the torus. We also used homogeneous data and measurements. The detailed results are presented by Schmitt et al. (2001b).

We found that both Seyfert 1's and Seyfert 2's have similar $60 \mu \mathrm{m}$ luminosities and $3.6 \mathrm{~cm}$ radio powers, as well as similar distributions of morphological types. The comparison between the host galaxy inclinations shows that there is a deficiency of Seyfert 1's in edge-on galaxies, which was known from previous studies and apparently contradicts the Unified Model. However, the model can be reconciled with the observations if we assume that Seyfert 1's observed edge-on will have their nucleus hidden by gas and dust in the galaxy disk. The extension of the radio emission in Seyfert 1's is, on average, smaller than in Seyfert 2's, as expected from the model. We also show that there is a similar percentage of Seyfert 1's and Seyfert 2's with companions.

These results, taken together, give strong support to the Unified Model. This indicates that previous results, which found differences in isotropic properties of Seyfert 1's and Seyfert 2's, were most likely due to selection effects.

Acknowledgments. Support for this work was provided by NASA grant AR-8383.01-97A. The National Radio Astronomy Observatory is a facility of the National Science Foundation operated under cooperative agreement by Associated Universities, Inc.

\section{References}

Antonucci, R. R. J. 1993, ARA\&A, 31, 473

Antonucci, R. R. J. \& Miller, J. S. 1985, ApJ, 297, 621

Bushouse, H. A. 1986, AJ, 91, 255

Dahari, O. 1984, AJ, 89, 966

de Grijp, M. H. K. et al. 1992, A\&AS, 96, 389

Dultzin-Hacyan, D. et al. 1999, ApJ, 513, L111

Fuentes-Williams, T. \& Stocke, J. T. 1988, AJ, 96, 1235

Gunn, J. 1979, in Active Galactic Nuclei, edited by C. Hazard \& S. Mitton, (Cambridge University Press, Cambridge), p.213

Hernquist, L. 1989, Nature, 640, 687

Keel, W. C. 1980, AJ, 85,198

Kinney, A. L. et al. 2000, ApJ, 537, 152

Laurikainen, E. \& Salo, H. 1995, A\&A, 293, 683

Lawrence, A. \& Elvis, M. 1982, ApJ, 256, 410

Maiolino, R. \& Rieke, G. H. 1995, ApJ, 454, 95

Malkan, M. A., Gorjian, V. \& Tam, R. 1998, ApJS, 117, 25

Pier, E. A. \& Krolik, J. H. 1992, ApJ, 401, 99

Pogge, R. W. 1989, ApJ, 345, 730

Rafanelli, P., Violato, M. \& Baruffolo, A. 1995, AJ, 109, 1546

Schmitt, H. R. 2001, AJ, in press

Schmitt, H. R. \& Kinney, A. L. 1996, ApJ, 463, 498

Schmitt, H. R. et al. 2001a, ApJS, 132, 199

Schmitt, H. R. et al. 2001b, ApJ, 555, 663

Schwartz, M. 1981, ApJ, 247, 77

Ulvestad, J. S., \& Wilson, A. S. 1989, ApJ, 343, 659 